\title{
Beamforming of a Linear Array Applying PSO Algorithm with Restrictive Approach
}

\author{
Vinícius Ludwig-Barbosa, Edson R. Schlosser, Cleiton Lucatel, Renato Machado and Marcos V. T. Heckler
}

\begin{abstract}
This paper presents a four-element linear array composed of E-shaped microstrip antennas designed to switchedbeam application in ISM (Industrial, Scientific and Medical) radio band. Particle Swarm Optimization (PSO) algorithm is used to optimize four different sets of amplitude and progressive phase shift to achieve four distinct radiation patterns controlling the major lobe direction and sidelobe level. For this application, two restrictive approaches are presented for the implementation of PSO in order to guide the algorithm to feasible solutions.
\end{abstract}

Index Terms-microstrip antenna, linear array, beamforming, switched-beam.

\section{INTRODUCTION}

Population-based algorithms such as Genetic Algorithm (GA), Particle Swarm (PSO) and Invasion Weed Optimization (IWO) have large application in Electromagnetics [2]-[4]. Comparing GA with PSO, the PSO algorithm has a simpler implementation based on few equations which control and evaluate the optimization process.

The Particle Swarm algorithm has been used in several applications which employ beamforming or beamsteering for high performance communication systems. In [5], the particle swarm optimization applied for beamforming design of 10 , 14 and 20-element linear arrays is described. In [6], a PSO algorithm combined with Numerical Electromagnetics Code (NEC) to produce the design curves of optimized log-periodic dipole arrays is presented. The algorithm is also applied on an innovative method to reach optimal radiation pattern of adaptive linear arrays by using phase-only disturbance in [7]. An interesting approach is presented in [8], in which a broadband MEMS (microelectromechanical systems) RHCP/LHCP reconfigurable patch array using E-shaped elements is optimized by PSO and a $20 \% \mathrm{~S}_{11}$-AR bandwidth was obtained using this technique. $S_{11}$-AR bandwidth stands for the range of frequency in which $\mathrm{S}_{11} \leq-10 \mathrm{~dB}$ overlaps $\mathrm{AR} \leq 3 \mathrm{~dB}$. In [9] and [10], E-shaped patches are applied in a $2 \times 2$ array for digital TV signal in C-Band $(4.4-5.0 \mathrm{GHz})$. Eshaped antennas exhibited better performance when compared

The Associate Editors coordinating the review of this manuscript and approving it for publication were Profs. Cecílio Pimentel and Marcelo da Silva Pinho.

Vinícius Ludwig-Barbosa and Renato Machado are with Signal Processing and Communications Research Group (GPSCom), Universidade Federal de Santa Maria, RS, Brazil. E-mail: viniciuslbar@mail.ufsm.br. Edson R. Schlosser, Cleiton Lucatel and Marcos V. T. Heckler are with Universidade Federal do Pampa, Alegrete, RS, Brazil. E-mail: edsonschlosser@unipampa.edu.br.

A preliminary version of this paper was presented in XXXIII Simpósio Brasileiro de Telecomunicações (SBrT'15), Juiz de Fora, MG, Brazil, September 1-4, 2015 [1]

Digital Object Identifier: 10.14209/jcis.2016.11 to omnidirectional quarter-wave monopoles, and rectangular and triangular patches. E-shaped radiators are also applied in circular polarization such as in [11], in which a modified patch is developed for WLAN (Wireless Local Area Network) application.

This paper applies the PSO to synthesize four radiation patterns for a four-element array. The idea is to analyze the feasibility of this structure to work as a switched-beam system, so as to provide a nearly uniform power distribution in the azimuth plane and to control power radiation in undesired directions. In order to avoid bad convergence on the optimization, two approaches are presented to achieve the best performance of this antenna array. The former considers no restriction of minimum power while the latter takes this constraint into account. The importance of the latter approach is verified. An alternative approach for the minimum power restriction is described.

The paper is organized as follows. A brief discussion about the antenna array theory, E-shaped patch antenna concept and details about the antenna array modeling and construction are presented in Section II. A prototype of the array is also presented along with S-parameters and radiation pattern measurements. Further, the PSO algorithm is discussed in Section III, in which the main equations applied in this optimization method are shown. In addition, Section IV describes the parameters and approaches assumed for this implementation. Finally, Sections V and VI present the synthesized beams for the four-element antenna array and some final considerations, respectively.

\section{Four-ElEMENT ARRAY}

The antenna array has been designed to provide the coverage of a $60^{\circ}$ sectored cell. It is composed of E-shaped patch antennas and operates in the 2.4-GHz ISM (Industrial, Scientific and Medical) radio band, suitable for applications in wireless systems, such as WLAN (Wireless Local Area Network).

\section{A. Antenna Array Theory}

According to the antenna array theory, the total electric field radiated by a linear array, as shown in Figure 1, composed of $N$ elements placed along $x$-axis is given by

$$
\vec{E}_{\text {array }}=\sum_{i=1}^{N} a_{i} \vec{E}_{i}(\theta, \phi) e^{j k_{0} x_{i} \sin (\theta) \cos (\phi)},
$$

where $a_{i}$ is a complex value that stands for the excitation coefficient at the port of the $i$-th antenna, $\vec{E}_{i}$ is the electric field 
radiated by each array element, $x_{i}=(i-1) d$ is the location of the $i$-th array element on the $x$-axis, $d$ is the uniform adjacent spacing between elements, and $k_{0}$ is the propagation constant in free space [12]. The representation in (1) is the classical antenna array theory form, which is based on the assumption that the patterns of the individual elements are identical. In matrix form, (1) can be rewritten as

$$
\mathbf{E}_{\text {array }}(\theta, \phi)=\mathbf{a e}(\theta, \phi),
$$

where $\mathbf{a}$ and $\mathbf{e}(\theta, \phi)$ are vectors described by

$$
\mathbf{a}=\left[\left|I_{1}\right| \angle \delta_{1} \quad\left|I_{2}\right| \angle \delta_{2} \ldots\left|I_{N}\right| \angle \delta_{N}\right]
$$

and

$$
\begin{array}{r}
\mathbf{e}(\theta, \phi)=\left[\mathbf{e}_{1}(\theta, \phi) e^{j \psi_{1}} \mathbf{e}_{2}(\theta, \phi) e^{j \psi_{2}}\right. \\
\left.\ldots \mathbf{e}_{N}(\theta, \phi) e^{j \psi_{N}}\right]^{T},
\end{array}
$$

where the symbol $[\cdot]^{T}$ indicates the transpose of a $N$-element vector. In (3), $\left|I_{i}\right|$ and $\delta_{i}$ stand for the amplitude and phase of the excitation coefficients applied at the terminals of the $i$-th array element. In applications with beamforming and sidelobe level control, the a vector can be rewritten as

$$
\mathbf{a}=\left[\left|I_{1}\right| \angle 0\left|I_{2}\right| \angle \beta \ldots\left|I_{N}\right| \angle(N-1) \beta\right],
$$

where $\beta$ is the progressive phase shift between adjacent antennas and it is given as

$$
\beta=-k_{o} d \sin \theta \cos \phi_{o p t} \quad \text { (radians), }
$$

with $\phi_{\text {opt }}$ standing for the direction where the main beam should be pointed to.

An inherent error is produced on the pointing direction when $\beta$ is calculated analytically, since this assumption is correct only in the case of an array composed of isotropic elements. When real radiators are considered, this value should be treated as a rough estimation.

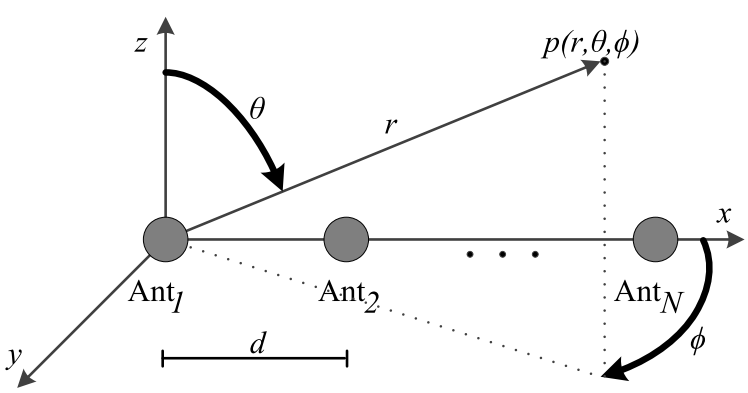

Fig. 1: Linear antenna array along the $x$-axis.

\section{B. E-shaped Patch Antenna}

A standard microstrip antenna design consists of one element (patch) that radiates electromagnetic waves. An electrically thin layer is present so as to separate the ground plane from the patch, which may exhibit theoretically any geometric shape. However, the canonical shapes, such as rectangle, ellipse, circle and triangle, are the most frequently used cases in microstrip antenna design [12]. The main drawback of canonical patch shapes is the narrow operating band.
Various techniques have been developed to enable the operation with a wider bandwidth than canonical geometries, such as the E-shaped patch [13]. This sort of antenna has a rectangular geometry with two parallel slits, which produce the excitation of two modes of operation. For the antenna in the upper operating mode (highest frequency), the slits have minor influence on the current density. Thus, only the dimensions of the central part of the antenna are responsible for most of the radiated energy. In the lower frequency mode, the slits start to contribute significantly in the behavior of the current density, modifying the way it flows on the patch. These operational characteristics guarantee that broad bandwidths are achieved easily.

\section{Array Design}

The designed array is composed of four elements placed along the $x$-axis. The interelement spacing (center-to-center) is $0.5 \lambda_{0}$. Such geometry has been adopted in order to produce a beam steering in the azimuth plane $\left(\theta=90^{\circ}\right)$. Figure 2 depicts the array structure along with information about dimensions and orientation. The array structure was optimized to provide

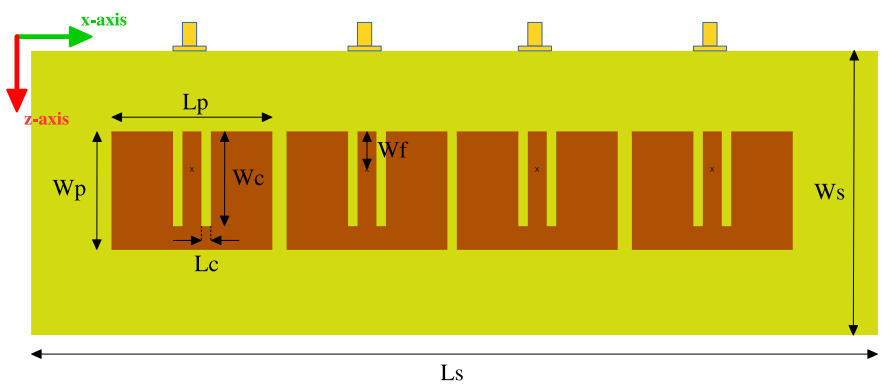

(a) Top View (all dimensions in millimeters): $\mathrm{L}_{p}=58.206, \mathrm{~W}_{p}=41.486$, $\mathrm{L}_{c}=3.781, \mathrm{~W}_{c}=33.499, \mathrm{~W}_{f}=11.8, \mathrm{~L}_{s}=298.706$ and $\mathrm{W}_{s}=90$.

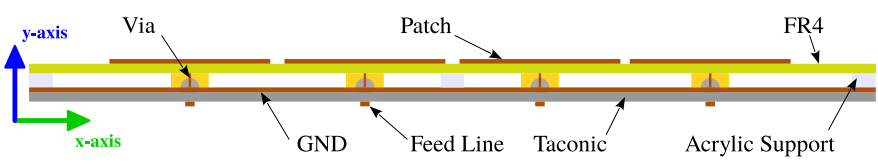

(b) Side View: stack-up.

Fig. 2: Four-element linear array composed of E-shaped patches: structure details.

a bandwidth of $8.3 \%$ at $2.4 \mathrm{GHz}$, which makes it suitable for different standards of wireless communication systems. Figure 2(a) shows the optimized top view structure dimensions. Each patch is fed by a $50 \Omega$ microstrip line and a cylindrical metallic via is used to connect them, as depicted in Figure 2(b).

A prototype has been built in order to validate the computational model done in HFSS [14] and is depicted in Figure 3. The whole structure is composed of a FR4-Epoxy laminate which contains the patches, an air layer and a Taconic TLC-338 substrate in which the feeding lines are impressed. The feeding substrate height is $1.524 \mathrm{~mm}$ while the antenna laminate is $1.54 \mathrm{~mm}$. The thickness of the air layer is $4.8 \mathrm{~mm}$. This is the same height of each acrylic support with length of $10 \mathrm{~mm}$. The material characteristics adopted for 


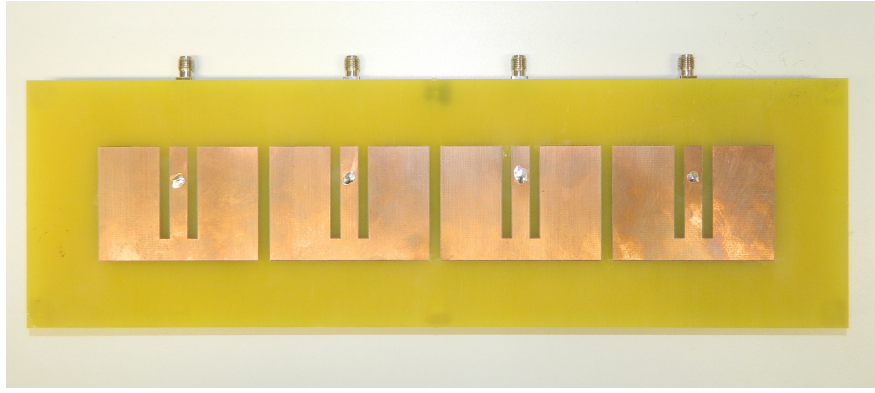

Fig. 3: Prototype implemented in the Antennas and Microwave Laboratory at UNIPAMPA.

this simulation are: $\varepsilon_{r_{\text {acrylic }}}=2.55, \tan \delta_{\text {acrylic }}=0.009$; $\varepsilon_{r_{F R 4}}=4.4, \tan \delta_{F R 4}=0.02, \varepsilon_{r_{\text {Taconic }}}=3.54$ and $\tan \delta_{\text {Taconic }}=0.0034$ [15], [16].

A slight deviation in frequency was observed in the measurements compared to the simulated results, which is approximately $6.5 \mathrm{MHz}$. Additionally, the measured bandwidth for the prototype is equal to $192 \mathrm{MHz}, 8 \mathrm{MHz}$ below the numerically predicted bandwidth. However, the prototype results are coherent to the simulation and validate the antenna array design. The variation between simulated and measured results was already expected due to inherent variation added on the model by the manufacture process. The S-parameters for each array element are depicted in Figure 4.

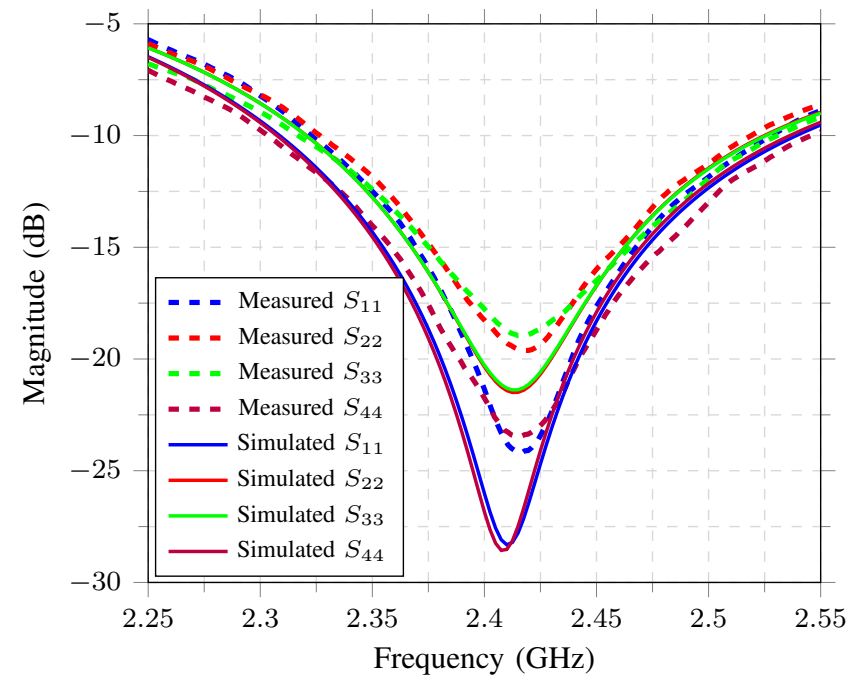

Fig. 4: S-parameters for each array element: the bandwidth is approximately $192 \mathrm{MHz}$ considering $|\mathrm{S}| \leq-10 \mathrm{~dB}$.

The gain patterns in the $\mathrm{H}$ and E-planes are depicted in Figure 5. These plots assume uniform excitation of $\mathrm{I}_{i}=1 \angle 0^{\circ} \mathrm{A}$ in each antenna array element. The result for this case is a broadside pattern in the H-plane, reaching maximum gain of $12.98 \mathrm{dBi}$. A tilted pattern is observed in the E-plane, which is a consequence of the asymmetric shape of the patch in this plane.

The radiation patterns of each array element were measured in a nearfield test facility [17]. The results are depicted in Figure 6. The simulated (HFSS) and measured (Nearfield)

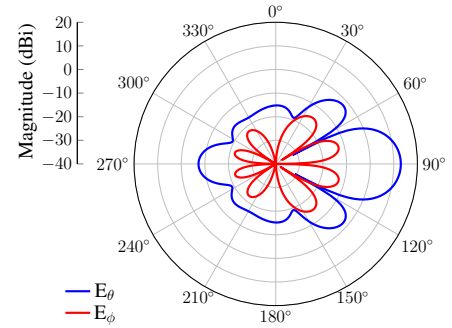

(a) H-plane $\left(\theta=90^{\circ}\right)$

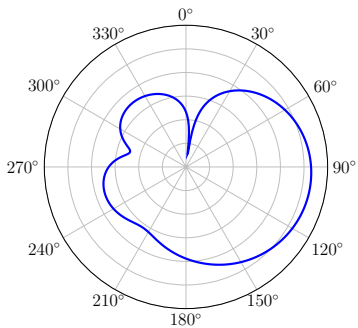

(b) E-plane $\left(\phi=90^{\circ}\right)$
Fig. 5: Simulated gain pattern for the four-element array: (a) H-plane; (b) E-plane.

radiation patterns present satisfactory match in the angular region between $0^{\circ}$ and $180^{\circ}$, which corresponds to the region where the PSO will be applied to perform the array synthesis.

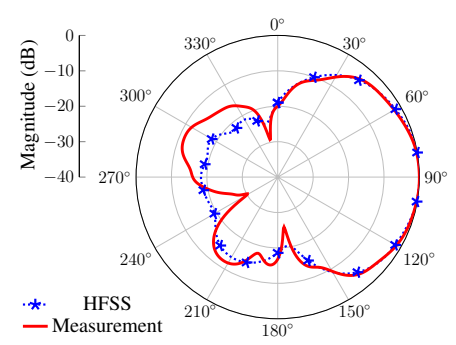

(a) Element \#1

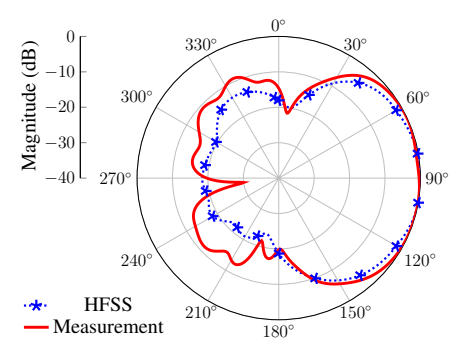

(c) Element \#3

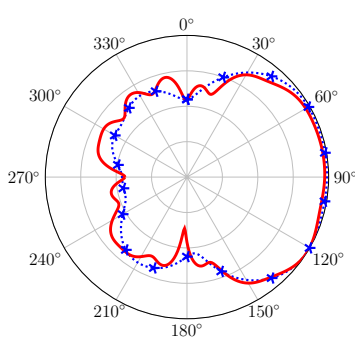

(b) Element \#2

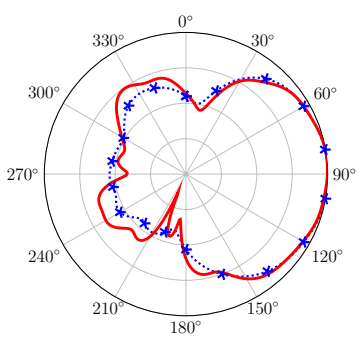

(d) Element \#4
Fig. 6: Comparison between simulated and measured radiation pattern for each array element in the azimuthal plane.

\section{Particle Swarm Optimization}

The Particle Swarm Optimization (PSO) is an advanced computational technique based on the movement of a swarm in order to find the best solution defined by the fitness function during successive iterations. The analogy of a bee swarm searching for the highest concentration of flowers in a garden perfectly fits the idea of this optimization method [3]. The implementation of this algorithm is mainly dependent on three initial definitions. First, one needs to define which parameters shall be optimized, such as complex excitation coefficients or even the array geometry [18]-[20]. Next, the range of possible solutions must be determined. And finally, the fitness function must be defined, which is used to evaluate the solutions at every iteration. These parameters should be chosen carefully, since they can vary depending on the application. 
The algorithm is started by setting up the velocity and location of each particle randomly. In the first iteration, the fitness function evaluates each particle position. A score is defined for each dimension (assuming an $n$-dimensional problem). The calculated score is set as its particle best solution $\left(p_{\text {best }}\right)$. The best score among the swarm is set as global best solution $\left(g_{\text {best }}\right)$.

In order to explore the searching space, the first step is to perform a systematic motion in each particle. This motion is based on the particle position in relation to its best solution and to the swarm best solution at the current iteration. Equation (7) computes the velocity applied to the particle to induce the motion in the next iteration as

$$
\begin{gathered}
\vec{v}_{n+1}^{k}=w \vec{v}_{n}^{k}+C_{1} \operatorname{rand}(\cdot)\left(p_{\text {best }}^{k}-p_{n}^{k}\right) \hat{u}+\ldots \\
C_{2} \operatorname{rand}(\cdot)\left(g_{\text {best }}-p_{n}^{k}\right) \hat{a},
\end{gathered}
$$

where $\vec{v}_{n}^{k}$ and $\vec{v}_{n+1}^{k}$ are the current and the next velocity vectors, $p_{n}^{k}$ is the current vector position of the $k$-th particle in the $n$-th iteration, $\hat{u}$ is a unit vector pointing from $p_{n}^{k}$ to $p_{\text {best }}^{k}$ and $\hat{a}$ is a unit vector pointing from $p_{n}^{k}$ to $g_{b e s t}$. These variables are illustrated in Figure 7 for the case of a two-dimensional searching space. Further, $w$ is the inertia coefficient, $C_{1}$ and $C_{2}$ are two positive numbers (cognitive and social acceleration constants). The function $\operatorname{rand}(\cdot)$ generates a random number within 0 and 1 and it models the arbitrary movement of the swarm.

By analyzing (7), it is possible to conclude that the particle is attracted by its best memory $\left(p_{\text {best }}\right)$ and by social influence of the swarm $\left(g_{b e s t}\right)$. The intensity of this pull is given by $C_{1} \operatorname{rand}(\cdot)$ and $C_{2} \operatorname{rand}(\cdot)$. The farthest is the position from $p_{\text {best }}$ and $g_{\text {best }}$, the stronger is the pull and the faster is the acceleration towards these positions. The closer is the position, the weaker is the pull and the lower is the acceleration towards these positions. The inertia coefficient $(w)$ stands for a factor to keep the original particle on its own path. Assuming all velocities updated, the next step is to determine the position of each particle in the next iteration based on

$$
p_{n+1}^{k}=p_{n}^{k}+\vec{v}_{n}^{k}
$$

In the optimization of an antenna array with PSO, a particle stands for a potential set of coefficients that synthesize the desired radiation pattern. In matrix form, (7) and (8) are rewritten as

$$
\begin{gathered}
\mathbf{V}_{n+1}^{k}=w \mathbf{V}_{n}^{k}+C_{1} \operatorname{rand}(\cdot)\left(\mathbf{P}_{\text {best }}^{k}-\mathbf{P}_{n}^{k}\right) \hat{u}+\ldots \\
C_{2} \operatorname{rand}(\cdot)\left(\mathbf{G}_{\text {best }}-\mathbf{P}_{n}^{k}\right) \hat{a} \\
\mathbf{P}_{n+1}^{k}=\mathbf{P}_{n}^{k}+\mathbf{V}_{n+1}^{k},
\end{gathered}
$$

where $\mathbf{P}_{n}^{k}$ and $\mathbf{V}_{n}^{k}$ are vectors described, for this application, by

$$
\begin{aligned}
& \mathbf{P}_{n}^{k}=\left[\begin{array}{ll}
\left|I_{1}\right| & \left|I_{2}\right| \ldots\left|I_{N}\right| \quad \beta
\end{array}\right], \\
& \mathbf{V}_{n}^{k}=\left[\begin{array}{lll}
\Delta I_{1} & \Delta I_{2} \ldots \Delta I_{N} & \Delta \beta
\end{array}\right] .
\end{aligned}
$$

Finally, the algorithm returns to the fitness evaluation. Whenever a best $\mathbf{P}_{\text {best }}$ or $\mathbf{G}_{\text {best }}$ is reached, their scores and locations are updated. The whole process repeats until either a particle fits the requirements tested by the fitness function or a maximum number of iterations is reached. After the iterative process, the values of the $\mathbf{G}_{\text {best }}$ should be applied to (5) and combined to (4), resulting in the array radiation pattern given in (2).

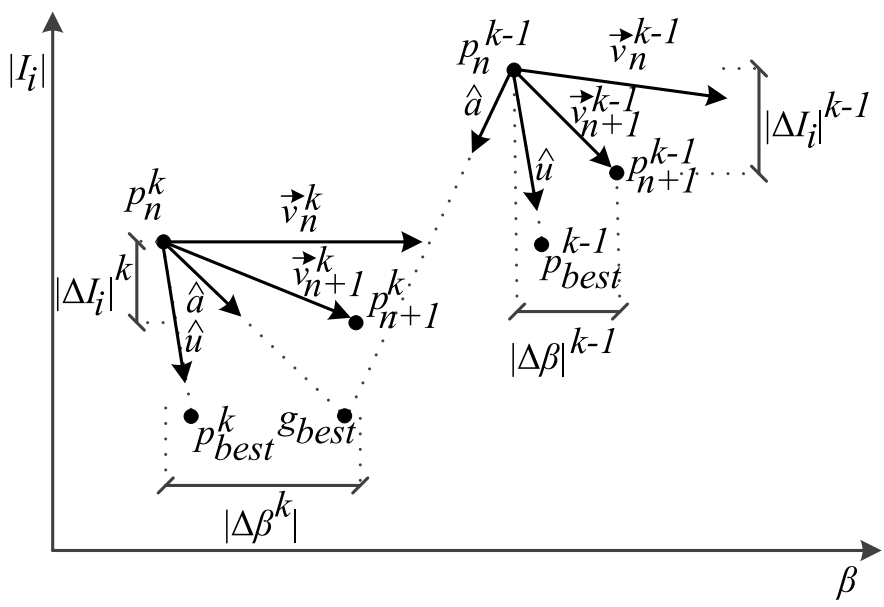

Fig. 7: Displacement of a particle in a two-dimensional search space.

\section{SYNTHESIS OF THE ARRAY WITH SWITCHED-BEAMS}

The choice of the number of radiation patterns required for a switched-beam application takes into account the balance between stable performance during transmission and system requirements. One important feature is the number of available bits for feedback information used to choose the beam to establish the communication.

In an ideal system, a switched-beam transmitter would provide as many beams as necessary in order to yield uniform radiation power to a service user inside a restricted sector of coverage. However, in a real-world scenario, there is a feedback channel between the service user and the transmitter, which sends only a limited length of bits to define the beam to be applied during a transmission frame. This means that the established channel is quantized and, consequently, there must be a compromise between the antenna array design, which includes the number of beams required, and the SNR (signalto-noise ratio) requirement. The number of beams will depend on the amount of bits transmitted on the feedback channel and is governed by the constraints of the system. For example, if the feedback channel supports $n$ bits, then the transmitter should be able to produce beams pointing to $2^{n}$ directions in the azimuthal plane.

The analysis presented in [21] proves that an amount of beams equivalent to the number of elements ( $n$ elements and $n$ beam-pointing directions) in a given antenna array is a good trade-off in terms of overall system costs and SNR performance. An amount of beams bigger than the number of elements causes small improvements in the system performance, tending to average SNR level saturation.

Consequently, four distinguished radiation patterns are required to yield efficient coverage when the antenna array designed is applied in a scenario of a $60^{\circ}$ sector. These radiation patterns should also have sidelobe levels up to $20 \mathrm{~dB}$ 
below the major lobe level to avoid undesirable interference. A schematic of this system is depicted in Figure 8. As expected for a switched-beam application, there is an inherent ripple of the radiation power along the azimuthal plane. The arrows show these ripples between the maximum (stars) and the minimum radiation power points (on the intersection of adjacent beams). Additionally, it is also presents the bit codes used in the feedback channel to set up the beam applied in each transmission frame.

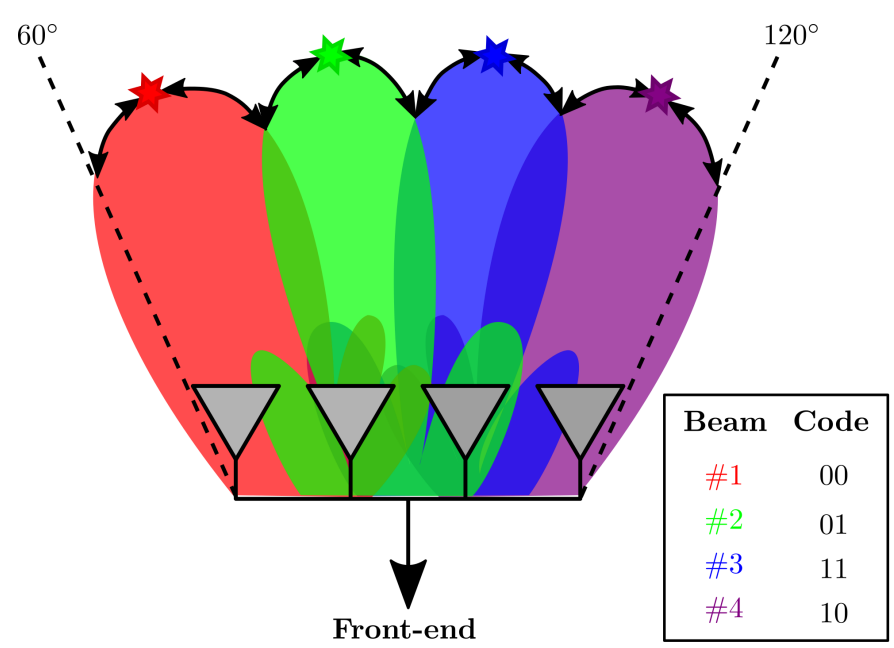

Fig. 8: Switched-beam sectored system composed by four radiation patterns.

The PSO algorithm has been implemented in order to synthesize those required radiation patterns. Its implementation considered twenty particles as possible solution for this specific problem. The position of each particle is given by a five-dimension space, in which four dimensions are related to the excitation amplitudes and one to the progressive phase shift. Each particle is evaluated by the fitness function at the beginning of every iteration. The maximum number of iterations is set to fifty. Thus, the optimal solution is reached either by fitting the established requirements or by the end of the fiftieth iteration. The fitness function applied for this problem is given by

$$
\text { fitness }=m_{1} \text { error }_{1}+m_{2} \text { error }_{2},
$$

where $m_{1}$ and $m_{2}$ are constant factors and

$$
\begin{gathered}
\text { error }_{1}=\sum_{q=1}^{Q}\left|\left(\mathrm{E}\left(\phi_{q}\right)-\mathrm{SLL}\right)\right| \forall \mathrm{E}\left(\phi_{q}\right)>\mathrm{SLL}, \\
\text { error }_{2}=\left|\phi_{\text {opt }}-\phi_{\max }\right|,
\end{gathered}
$$

where $Q$ is the total number of sidelobes in the radiation pattern under evaluation, $\phi_{\max }$ is the direction of the maximum radiation obtained and $\phi_{\text {opt }}$ is the requested direction.

The calculated values indicate how far such particle is from the current $\left(p_{\text {best }}\right)$ and $\left(g_{\text {best }}\right)$. Therefore, the bigger is the error, the worse is the solution. The scores are also important to update particle $\left(p_{\text {best }}\right)$ and global best $\left(g_{\text {best }}\right)$ solution.

Applying the invisible/reflecting walls approach [22], only particles inside the defined space of possible solutions are evaluated by the fitness function at each iteration. The particles which are out of boundaries have their velocities inverted, aiming to bring these particles back into the space of possibles solutions on the next iteration. The approach has the advantage of reducing the computational effort involved in the optimization, since only feasible solutions are evaluated. This boundary condition has been implemented on the first and second approaches (Sections V-A and V-B).

In some applications, for the sake of convergence, it is necessary to define the space of possible solutions carefully. Assuming normalized excitation coefficients, any value within 0 and 1 is theoretically valid and consequently a possible solution. This constraint is initially applied in the first approach.

Another important step is to define the parameters applied in (7). The inertia coefficient is linearly decreased throughout the optimization. Initially, the coefficient is set in 0.9 . The minimum possible value for the inertial coefficient is 0.4 at the 50-th iteration. This variation is adopted firstly to provide a global exploration of the solution space and gradually modify this behavior to perform a exploration around the best position of each particle and around the global solution [23]. The equation used to linearly decrease the inertia coefficient is

$$
w_{i}=w_{\max }-\left(w_{\max }-w_{\min }\right) \frac{i}{i_{\max }},
$$

where $w_{\max }=0.9, w_{\min }=0.4, i_{\max }$ is defined as 50 and $i$ is the $i$-th iteration. Coefficients $C_{1}$ and $C_{2}$ have been set to 0.5 for all the implemented approaches. Approaches $\mathrm{A}$ and $B$ also assume limited maximum velocity to the range of the excitation amplitudes space.

The progressive phase shift is optimized along with the excitation amplitudes. There is a computational advantage in optimizing only the progressive phase shift compared to optimizing the phase of each element since it reduces the total number of parameter involved in the process. Instead of $2 N$ parameters, $N$ amplitudes and $N$ phases, this procedure optimizes just $N+1$ variables: $N$ amplitudes and $\beta$. The progressive phase shift space has no limitation since trigonometric functions are periodic. Every particle assumes the progressive phase shift given in (6) only at the first iteration as an initial estimation. As previously mentioned, (6) is valid exclusively for isotropic antennas and produces a deviation when directly applied for arrays composed of antennas with different radiation characteristics [24]. Figure 9 illustrates this aspect. The farther from the broadside the beam should be steered to, the larger becomes the pointing error. This situation becomes clear for the case of pointing the major lobe to $\phi_{\max }=60^{\circ}$. Additionally, the presence of a grating lobe did not allow controlling the sidelobe level properly in the case.

The simulated radiation pattern of each array element considered in the optimization takes into account the mutual coupling induced by the nearby elements. In order to consider this effect during the pattern optimization, one element has been fed with unitary current whilst the feed currents for the others have been set to null. This is equivalent to a matched termination. This procedure was repeated for each antenna. The radiation patterns for each element in the $\mathrm{H}$ plane correspond to the four terms of $\mathbf{e}\left(90^{\circ}, \phi\right)$ vector in (2). 


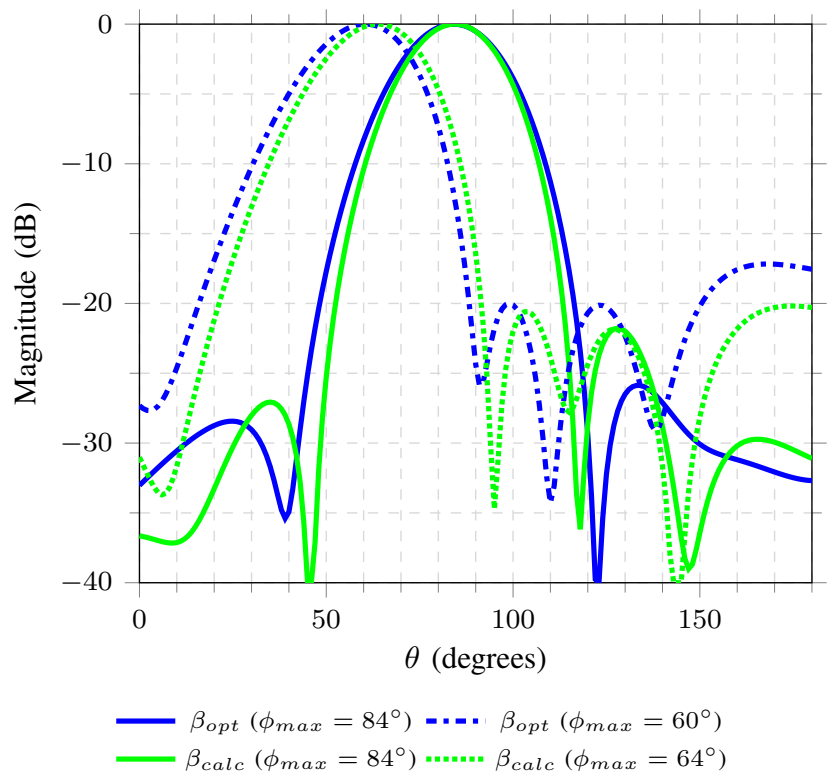

Fig. 9: Radiation patterns before and after optimization of the progressive phase shift.

Applying this methodology, the simulated radiation pattern of the antenna array considered in the optimization reproduces a practical behavior likewise the one observed on the prototype measurements.

\section{RESULTS}

The four sets of excitation coefficients have been synthesized for the directions given in Table I considering all the $\phi$ values in the $\mathrm{H}$-plane $\left(\theta=90^{\circ}\right)$.

TABLE I: Pointing directions for each of the four beams.

\begin{tabular}{|c||c|}
\hline Beamforming & $\phi$ \\
\hline \hline$\# 1$ & $72^{\circ}$ \\
\hline$\# 2$ & $84^{\circ}$ \\
\hline$\# 3$ & $96^{\circ}$ \\
\hline$\# 4$ & $108^{\circ}$ \\
\hline
\end{tabular}

An overview of the parameters applied in the Approach A is presented in Table II.

TABLE II: Parameter settings for Approach A.

\begin{tabular}{|c|c|}
\hline Particles & 20 \\
\hline Iteration limit & 50 \\
\hline SLL & $20 \mathrm{~dB}$ \\
\hline$w$ & {$\left[\begin{array}{ll}0.9 & 0.4\end{array}\right]$} \\
\hline $\mathrm{C}_{1}$ and $\mathrm{C}_{2}$ & 0.5 \\
\hline $\mathrm{m}_{1}$ and $\mathrm{m}_{2}$ & 50 \\
\hline Amplitudes & {$\left[\begin{array}{ll}0 & 1\end{array}\right]$} \\
\hline Velocities & {$\left[\begin{array}{ll}-1 & 1\end{array}\right]$} \\
\hline
\end{tabular}

These pointing directions have been chosen in order to obtain points of maximum radiation equally spaced. Considering a sector of $60^{\circ}$ (within $60^{\circ}$ and $120^{\circ}$ ), a step of $12^{\circ}$ was assumed for this scenario.
The excitation coefficients obtained using the PSO algorithm are presented in Table IV and the optimized progressive phase shifts are presented in Table V.

\section{A. Optimization without power constraints}

Analyzing the optimized amplitudes obtained without constraint of a minimum percentage of power in each array element, it is possible to state that only one or two elements have excitation amplitudes greater than $10 \%$ of power $(\sqrt{10 / 100}=0.316)$ in comparison to the largest value. These possible solutions are not of interest when an antenna array is considered for a specific application, since the main goal is to achieve as higher gain as possible and, consequently, to concentrate the radiation exclusively towards the directions of interest. In this application, elements with lower contribution have a more significant impact on the resultant radiation pattern, since the array is composed of only four elements. As a result, the main effect in the radiation pattern is a loss in directivity, as it is depicted in Figure 11.

\section{B. Optimization with power constraints}

In order to create a constraint on the PSO algorithm to avoid the solution presented in Section V-A, the search space has been modified. Previously, the feasible space of excitation amplitude was within 0 and 1 . Alternatively, each array element shall be fed by at least $10 \%$ of the possible maximum power. Throughout the optimization, whenever a particle has some of its amplitude excitation below this threshold value, this possible solution is not evaluated by the fitness function. In other words, the solution is discarded at the current iteration.

A parameter overview for Approach B implementation, regarding the modification in the space of solutions for amplitudes, is presented in Table III.

TABLE III: Parameter settings for Approach B

\begin{tabular}{|c|c|}
\hline Particles & 20 \\
\hline Iteration limit & 50 \\
\hline SLL & $20 \mathrm{~dB}$ \\
\hline$w$ & {$\left[\begin{array}{ll}0.9 & 0.4\end{array}\right]$} \\
\hline $\mathrm{C}_{1}$ and $\mathrm{C}_{2}$ & 0.5 \\
\hline $\mathrm{m}_{1}$ and $\mathrm{m}_{2}$ & 50 \\
\hline Amplitudes & {$\left[\begin{array}{ll}0.316 & 1.000\end{array}\right]$} \\
\hline Velocities & {$\left[\begin{array}{ll}-1 & 1\end{array}\right]$} \\
\hline
\end{tabular}

By considering the power constraint one can see in Table IV that all the elements present excitation amplitudes greater than the defined lower boundary. Consequently, it is possible to verify an improvement on the synthesized beams, as depicted in Figure 11. The main improvement when the minimum power constraint is taken into account is a greater directivity compared to the previous case. A major lobe with narrower width is achieved, which is a desirable trait for a four-element antenna array. In addition, the requirement of sidelobe level below $20 \mathrm{~dB}$ has been achieved. 


\section{Alternative approach}

Aiming to keep a minimum contribution from each element on the resultant radiation pattern and to avoid the necessity of setting a minimum power constraint, a restriction regarding the number of lobes is established. Thus, only solutions that yield radiation patterns with a minimum number of lobes are assumed to be acceptable. The restriction is set as

$$
n_{\text {lobes }}>n_{\text {elem }}-2 \text {, }
$$

where $n_{\text {lobes }}$ is the number of detected peaks, including the major lobe, and $n_{\text {elem }}$ is the number of antennas. This is shown as Approach C in Figure 11.

Additionally to the power restriction, the boundary conditions (reflective/invisible walls) were neglected and particles velocity was loosen in this implementation. These modifications have not affected the overall convergence and the synthesized radiation patterns are equivalent to the ones yielded by Approach B. This achievement is pointed as an advantage of Approach C over Approach B, since it skips the necessity of defining a minimum power for each application. Consequently, the parameters applied in Approach $\mathrm{C}$ are the same shown in Table II. A flowchart resumes the optimization steps in Figure 10.

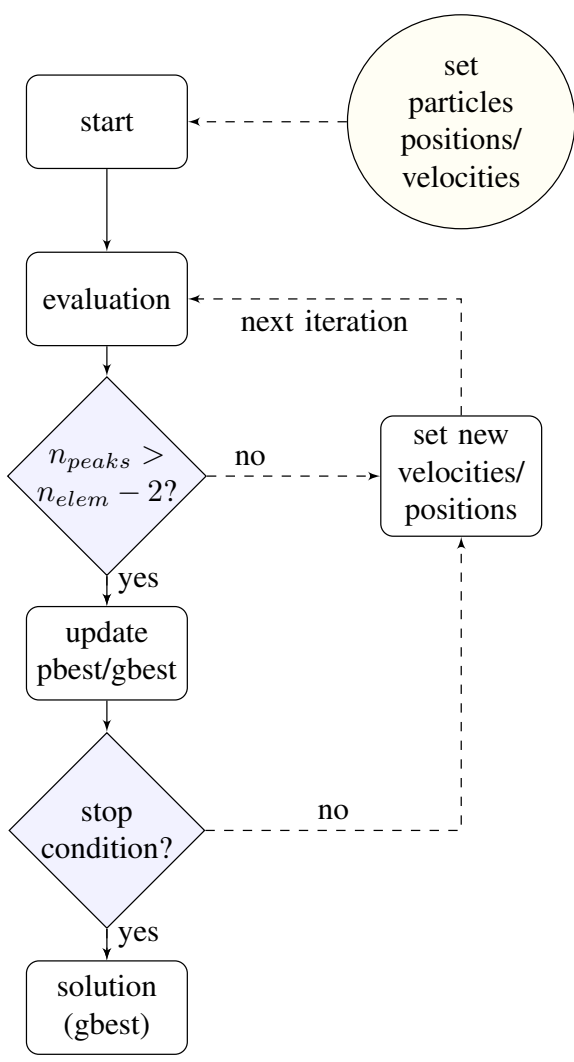

Fig. 10: PSO flowchart for the alternative approach.

For the sake of comparison, the HPBW (Half-Power Bandwidth) have been analyzed for the radiation patterns presented in Figure 11. Table VI summarizes the HPBW values for the optimized beams.

The results prove that Approach B achieved narrower beamwidth than Approach $\mathrm{A}$ in all pointing direction; $8^{\circ}$ in
TABLE IV: Amplitude of the excitation coefficients for each synthesized beam and for each approach.

\begin{tabular}{|c||c|c|c|c|}
\hline \multicolumn{1}{|c||}{} & \multicolumn{4}{c|}{ Beams } \\
\hline \multicolumn{1}{|c||}{ Without Power Constraints } \\
\hline Element & $\# 1$ & $\# 2$ & $\# 3$ & $\# 4$ \\
\hline \hline 1 & 0.1564 & 0.2748 & 0.2208 & 0.1211 \\
\hline 2 & 0.2716 & 0.4907 & 0.8690 & 0.2748 \\
\hline 3 & 0.4977 & 0.8655 & 0.3222 & 0.6336 \\
\hline 4 & 0.1984 & 0.2969 & 0.1682 & 0.2615 \\
\hline \multicolumn{1}{|c||}{ With } & Power & Constraints \\
\hline \hline 1 & 0.4255 & 0.4033 & 0.3635 & 0.4038 \\
\hline 2 & 0.7058 & 0.6842 & 0.7217 & 0.7058 \\
\hline 3 & 0.7566 & 0.6001 & 0.7288 & 0.7235 \\
\hline 4 & 0.3921 & 0.4289 & 0.5406 & 0.4928 \\
\hline \multicolumn{1}{|c||}{ Alternative Approach } \\
\hline \hline 1 & 0.4230 & 0.5097 & 0.4378 & 0.6122 \\
\hline 2 & 0.6590 & 0.7708 & 0.7013 & 0.9205 \\
\hline 3 & 0.6124 & 0.7926 & 0.8088 & 0.9030 \\
\hline 4 & 0.3040 & 0.3031 & 0.5776 & 0.5278 \\
\hline
\end{tabular}

TABLE V: Progressive phase shift for each synthesized beam and for each approach.

\begin{tabular}{|c|c|c|c|}
\hline \multicolumn{4}{|c|}{ Beamforming } \\
\hline \multicolumn{4}{|c|}{ Without Power Constraints } \\
\hline$\# 1$ & $\# 2$ & $\# 3$ & $\# 4$ \\
\hline \hline $59.36^{\circ}$ & $20.69^{\circ}$ & $-19.31^{\circ}$ & $-60.98^{\circ}$ \\
\hline \multicolumn{4}{|c|}{ With Power Constraints } \\
\hline \hline $61.37^{\circ}$ & $20.63^{\circ}$ & $-19.06^{\circ}$ & $-61.71^{\circ}$ \\
\hline \multicolumn{4}{|c|}{ Alternative Approach } \\
\hline \hline $61.75^{\circ}$ & $19.841^{\circ}$ & $-18.52^{\circ}$ & $-61.02^{\circ}$ \\
\hline
\end{tabular}

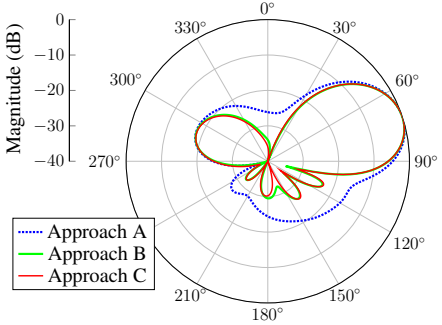

(a) Beam \#1

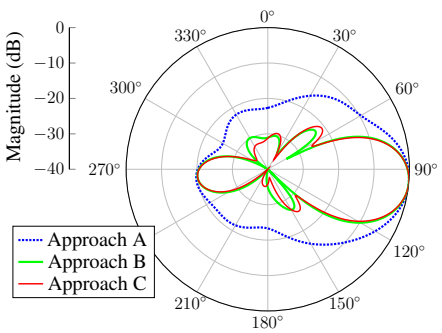

(c) Beam \#3

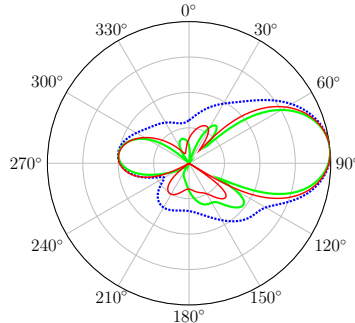

(b) Beam \#2

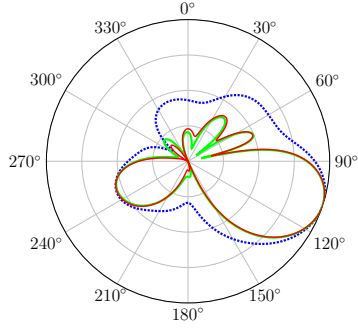

(d) Beam \#4
Fig. 11: Comparison of the radiation patterns for the three approaches considered in this paper.

best case and $2^{\circ}$ in worst case. In general, it is also possible to state that Approach $\mathrm{C}$ presents equivalent performance compared to Approach B. 
TABLE VI: HPBW values for each beam and for each approach.

\begin{tabular}{|c||c|c|c|}
\hline \multicolumn{1}{|c||}{} & \multicolumn{3}{c|}{ HPBW } \\
\hline Beam & Approach A & Approach B & Approach C \\
\hline \hline$\# 1$ & $30^{\circ}$ & $28^{\circ}$ & $28^{\circ}$ \\
\hline$\# 2$ & $30^{\circ}$ & $25^{\circ}$ & $28^{\circ}$ \\
\hline$\# 3$ & $34^{\circ}$ & $26^{\circ}$ & $25^{\circ}$ \\
\hline$\# 4$ & $31^{\circ}$ & $26^{\circ}$ & $26^{\circ}$ \\
\hline
\end{tabular}

\section{CONCLUSiOnS}

The PSO algorithm has been successfully applied in general problems, such as sidelobe level control for an array with four switched beams. Although, its implementation must consider some constraints in order to effectively achieve only feasible solutions. The implementation of PSO algorithm may converge in some cases to solutions in which the contribution of each element has no significant relevance to the overall radiation pattern, as it is depicted for the Approach A. In order to avoid these problems, two different approaches of PSO implementation were presented in this paper. The Approach C skips the necessity of defining a minimum amount of power per antenna and its solution is validated, since the resultant radiation patterns are quite similar to those achieved with Approach B.

Furthermore, a computational advantage is shown in this paper when the progressive phase shift strategy is considered, instead of optimizing absolute phases. This option reduces significantly the number of variables under optimization and its implementation is worthy in applications in which the array is composed of a large number of antennas.

\section{REFERENCES}

[1] V. Ludwig-Barbosa, M. P. Magalhães, E. R. Schlosser, R. Machado, and M. V. T. Heckler, "Beaforming of an E-shaped linear array for WLAN systems applying PSO algorithm with restrictive approach," in XXXIII Simpósio Brasileiro de Telecomunicações (SBrT'15). Brazil, Juiz de Fora, MG: SBRT, 2015, pp. 1-5.

[2] J. M. Johnson and Y. Rahmat-Samii, "Genetic algorithm optimization and its application to antenna design," in IEEE Antennas and Propagation Society International Symposium, vol. 01. IEEE, 1994. doi: http://dx.doi.org/10.1109/APS.1994.407746 pp. 326-329.

[3] J. Robinson and Y. Rahmat-Samii, "Particle swarm optimization in electromagnetics," Antennas and Propagation, IEEE Transactions on, vol. 52, no. 2, pp. 397-407, 2004. doi: http://dx.doi.org/10.1109/TAP.2004.823969

[4] S. Karimkashi and A. A. Kishk, "Invasive weed optimization and its features in electromagnetics," Antennas and Propagation, IEEE Transactions on, vol. 58, no. 4, pp. 1269-1278, 2010. doi: http://dx.doi.org/10.1109/TAP.2010.2041163

[5] G. Ram, R. Bera, D. Mandal, R. Kar, and S. P. Ghosal, "Novel particle swarm optimization based hyper beamforming of linear antenna arrays," in Students' Technology Symposium (TechSym), 2014 IEEE, 2014. doi: http://dx.doi.org/10.1109/TechSym.2014.6807931 pp. 148-153.

[6] M. Fernandez Pantoja, A. R. Bretones, F. Garcia Ruiz, S. G. Garcia, and R. G. Martin, "Particle swarm optimization in antenna design: Optimization of log-periodic dipole arrays," Antennas and Propagation Magazine, IEEE, vol. 49, no. 4, pp. 34-47, 2007. doi: http://dx.doi.org/10.1109/MAP.2007.4385594

[7] C.-H. Hsu, C.-H. Chen, W.-J. Shyr, K.-H. Kuo, Y.-N. Chung, and T.-C Lin, "Optimizing beam pattern of linear adaptive phase array antenna based on particle swarm optimization," in Genetic and Evolutionary Computing (ICGEC), 2010 Fourth International Conference on, 2010. doi: http://dx.doi.org/10.1109/ICGEC.2010.150 pp. 586-589.
[8] J. M. Kovitz, H. Rajagopalan, and Y. Rahmat-Samii, "Design and implementation of broadband MEMS RHCP/LHCP reconfigurable arrays using rotated E-shaped patch elements," Antennas and Propagation, IEEE Transactions on, vol. 63, no. 6, pp. 2497 - 2507, 2015. doi: http://dx.doi.org/10.1109/TAP.2015.2417892

[9] D. Wolansky and Z. Raida, "Antenna array of E-shaped patches for indoor receiving of the digital TV signal," in Electromagnetics in Advanced Applications (ICEAA), 2011 International Conference on, 2011. doi: http://dx.doi.org/10.1109/ICEAA.2011.6046441 pp. 757-760.

[10] S. Nagaraju, B. V. Kadam, L. J. Gudino, S. M. Nagaraja, and N. Dave, "Performance analysis of rectangular, triangular and E-shaped microstrip patch antenna arrays for wireless sensor networks," in Computer and Communication Technology (ICCCT), 2014 International Conference on, 2014. doi: http://dx.doi.org/10.1109/ICCCT.2014.7001494 pp. 211215.

[11] A. Khidre, K. F. Lee, F. Yang, and A. Elsherbeni, "Wideband circularly polarized E-shaped patch antenna for wireless applications [wireless corner]," Antennas and Propagation Magazine, IEEE, vol. 52, no. 5, pp. 219-229, 2010. doi: http://dx.doi.org/10.1109/MAP.2010.5687547

[12] C. A. Balanis, Antenna Theory Analysis and Design, 3rd ed. WilleyInterscience, 2005.

[13] F. Yang, X.-X. Zhang, X. Ye, and Y. Rahmat-Samii, "Wide-band Eshaped patch antennas for wireless communications," Antennas and Propagation, IEEE Transactions on, vol. 49, no. 7, pp. 1094-1100, Jul. 2001. doi: http://dx.doi.org/10.1109/8.933489

[14] ANSYS Corp., "ANSYS HFSS version 15 - User's guide," 2013.

[15] Kaysons-Akrylik Furniture \& Accessories, "Physical properties of acrylic sheets," 2005.

[16] Taconic-Add, "Advanced PCB materials: Product selection guide," 2014

[17] A. D. Yaghjian, "An overview of near-field antenna measurements," IEEE Transactions on Antennas and Propagation, vol. AP-34, no. 1, pp. 612-619, 1988. doi: http://dx.doi.org/10.1109/TAP.1986.1143727

[18] M. Khodier and M. Al-Aqil, "Design and optimisation of yagi-uda antenna arrays," Microwaves, Antennas Propagation, IET, vol. 4, no. 4, pp. 426-436, 2010. doi: http://dx.doi.org/10.1049/iet-map.2009.0054

[19] J. R. Perez Lopez and J. Basterrechea Verdeja, "Synthesis of linear arrays using particle swarm optimisation," in Antennas and Propagation, 2006. EuCAP 2006. First European Conference on, Nov. 2006. doi: http://dx.doi.org/10.1109/EUCAP.2006.4584909 pp. 1-6.

[20] S. K. Singh, V. B. Chandrudu, and G. K. Mahanti, "Synthesis of linear array antenna for fixed level of side lobe level and first null beam width using particle swarm optimization," in Communications and Signal Processing (ICCSP), 2013 International Conference on, 2013. doi: http://dx.doi.org/10.1109/iccsp.2013.6577058 pp. 275-279.

[21] V. Ludwig-Barbosa, E. Schlosser, R. Machado, F. G. Ferreira, S. M. Tolfo, and M. V. T. Heckler, "Linear array design with switched beams for wireless communications systems," in International Journal of Antenna and Propagation, 2014. doi: http://dx.doi.org/10.1155/2015/278160 pp. 1-9.

[22] S. Xu and Y. Rahmat-Samii, "Boundary conditions in particle swarm optimization revisited," Antennas and Propagation, IEEE Transactions on, vol. 55, no. 3, pp. 760-765, 2007. doi: http://dx.doi.org/10.1109/TAP.2007.891562

[23] R.C. Eberhart and Y. Shi, "Evolving artificial neural networks," in Proc. 1998 Int. Conf. Neural Networks and Brain, Beijing, P.R.C., 1998.

[24] E. R. Schlosser, R. L. Farias, M. V. T. Heckler, and R. Machado, "Optimization of switched-beam arrays for communication systems," in 2014 11th International Symposium on Wireless Communications Systems (ISWCS). Barcelona, Spain: IEEE, Aug. 2014. doi: 10.1109/ISWCS.2014.6933420 pp. 579-583.

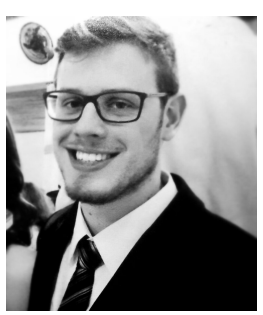

Vinícius Ludwig-Barbosa was born in Rio Grande do Sul, Brazil. He received the B.Sc. in Electrical Engineering from Universidade Federal de Santa Maria (UFSM), Santa Maria, Brazil, in 2013. Since 2012, he has joined the Signal Processing and Communications Research Group (GPSCom) and currently is a master student at UFSM. His research interests are beamforming optimization methods, antennas \& propagation and digital beamforming. 


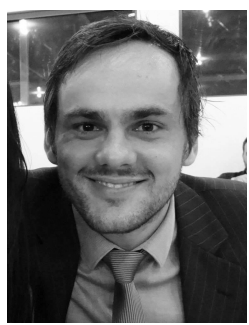

Edson R. Schlosser was born in Santa Rosa, Brazil, in 1989. He received the B.S. and M.Sc. degree in electrical engineering from Universidade Federal do Pampa (UNIPAMPA), Alegrete, Brazil, in 2011 and 2014. Since 2013, he has been an teaching assistant at Universidade Federal do Pampa, Alegrete, Brazil and currently is a member of Electronic Systems Research Group (GPSel). His research interest includes the development of antenna arrays for several applications, microstrip devices for microwave and forming radiation pattern optimization methods for beamshaping and beam-

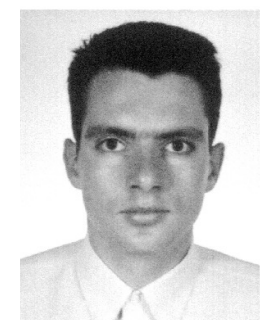

Cleiton Lucatel was born in Chapecó, Brazil, in 1982. He received the technical degree in Electromechanical (2001) from Serviço Nacional de Aprendizagem Industrial - SENAI, Chapecó, Brazil, and the technical degree in Electroelectronics (2008) from Centro Federal de Educação Tecnológica CEFET, Chapecó, Brazil, and the technical degree in Industrial Automation (2012) from Colégio Técnico Industrial de Santa Maria - CTISM, Santa Maria, Brazil. He has been working as a member of the technical staff at UNIPAMPA since 2012.

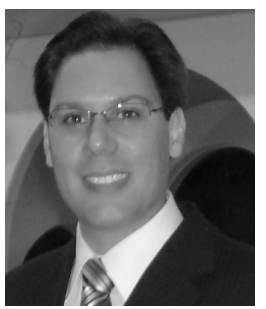

Renato Machado (S'04-M'08) received the B.S.E.E. degree from Universidade Estadual de São Paulo (UNESP), Ilha Solteira, SP, Brazil, in 2001. He received the M.Sc. degree and the Ph.D. degree in electrical engineering from Universidade Federal de Santa Catarina (UFSC), Florianópolis, SC, Brazil, in 2004 and 2008, respectively. He was a visiting researcher in the Department of Electrical Engineering, Arizona State University (ASU), Tempe, AZ, U.S.A., from August 2006 to June 2007. He was a research engineer at Nokia Institute of Technology, Brazil, from October 2007 to March 2008, and a visiting Professor in the Department of Electrical Engineering, Universidade Federal de Juiz de Fora, MG, Brazil, from September 2008 to August 2009. From Nov. 2013 to Fev. 2015 Dr. Machado was a visiting professor at Blekinge Institute of Technology (BTH), developing a research project in partnership with Saab AB. Since August 2009, he has been a Professor at Universidade Federal de Santa Maria, RS, Brazil. His research interests include MIMO systems, space-time coding, cooperative communication, wireless communications, SAR systems and power line communications. Dr. Machado is a member of the IEEE Communications Society, and the Brazilian Telecommunications Society.

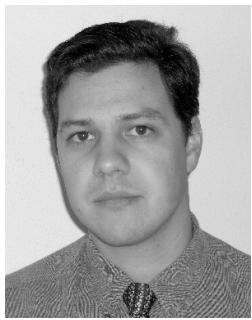

Marcos V. T. Heckler (M'06) and was born in Rio Grande, Brazil, in 1978. He received the BSc. degree in Electrical Engineering (Emphasis in Electronics) in 2001 from Universidade Federal de Santa Maria (UFSM), Brazil, the MSc. degree in Electronic Engineering (Microwaves and Optoelectronics) in 2003 from Instituto Tecnológico de Aeronáutica (ITA), Brazil, and the Dr.-Ing. degree in Electrical Engineering in 2010 from Technische Universität München, Germany. From April to August 2003 he worked as a Research Assistant with the Antennas and Propagation Laboratory at ITA, Brazil. From October 2003 to June 2010 he worked as a Research Associate towards his $\mathrm{PhD}$ with the Antenna Group, Institute of Communications and Navigation, German Aerospace Center (DLR). He is currently a Professor at Universidade Federal do Pampa, in Alegrete, Brazil. His current research interests are the design of microstrip antennas and arrays, and the development of numerical techniques for conformal microstrip antennas. 